\title{
Joint hyponormality of rational Toeplitz pairs
}

\author{
In Sung Hwang and Woo Young Lee
}

\begin{abstract}
We characterize hyponormal "rational" Toeplitz pairs which are pairs of Toeplitz operators whose symbols are rational functions in $L^{\infty}$. The main result of this article is as follows. If $\mathbf{T}=\left(T_{\phi}, T_{\psi}\right)$ is a hyponormal rational Toeplitz pair then $\phi-\beta \psi \in H^{2}$ for some constant $\beta$; in other words, their co-analytic parts necessarily coincide up to a constant multiple. As a corollary we get a complete characterization of hyponormal rational Toeplitz pairs.
\end{abstract}

Mathematics Subject Classification (2000). Primary 47B20, 47B35, 47A13.

Keywords. Hyponormal, jointly hyponormal, Toeplitz operators, Hankel operators, bounded type symbols, rational symbols.

\section{Introduction}

In the monograph [CL], R. Curto and the second named author characterized the joint hyponormality of Toeplitz pairs which are pairs of Toeplitz operators with trigonometric polynomial symbols. We here obtain a complete characterization of hyponormal rational Toeplitz pairs which are pairs of Toeplitz operators whose symbols are rational functions in $L^{\infty}$.

The Bram-Halmos criterion on subnormality ([Br] ) states that an operator $T$ on a Hilbert space $\mathcal{H}$ is subnormal if and only if $\sum_{i, j}\left(T^{i} x_{j}, T^{j} x_{i}\right) \geq 0$ for all finite collections $x_{0}, x_{1}, \cdots, x_{k} \in \mathcal{H}$. It is easy to see that this is equivalent to the following positivity test:

$$
\left(\begin{array}{cccc}
I & T^{*} & \ldots & T^{* k} \\
T & T^{*} T & \ldots & T^{* k} T \\
\vdots & \vdots & \ddots & \vdots \\
T^{k} & T^{*} T^{k} & \ldots & T^{* k} T^{k}
\end{array}\right) \geq 0 \quad(\text { all } k \geq 1)
$$

The second author was supported by a grant (KRF-2008-314-C00014) from the Korea Research Foundation. 
In 1988, the notion of "joint hyponormality" (for the general case of $n$-tuples of operators) was first formally introduced by A. Athavale [At]. He conceived joint hyponormality as a notion at least as strong as requiring that the linear span of the operator coordinates consist of hyponormal operators, the latter notion being called weak joint hyponormality.

Subnormality, joint hyponormality, and weak joint hyponormality have been studied by A. Athavale [At], J. Conway and W. Szymanski [CS], R. Curto [Cu], R. Curto, P. Muhly, and J. Xia [CMX], R. Douglas, V. Paulsen, and K. Yan [DPY], R. Douglas and K. Yan [DY], D. Farenick and R. McEachin [FM], C. Gu [Gu], S. McCullough and V. Paulsen [McCP], D. Xia [Xi], R. Curto and the second named author [CL], and others. Joint hyponormality originated from questions about commuting normal extensions of commuting operators, and it has also been considered with an aim at understanding the gap between hyponormality and subnormality for single operators. To date, much of the research on joint hyponormality has dealt with commuting tuples of hyponormal operators.

The study of jointly hyponormal Toeplitz operators started from D. Farenick and R. McEachin [FM]. They studied operators that form hyponormal pairs in the presence of the unilateral shift. Since the unilateral shift is a Toeplitz operator on the Hardy space of the unit circle, one can ask whether the results in [FM] extend to Toeplitz pairs, that is, pairs whose coordinates are Toeplitz operators on the Hardy space of the unit circle. R. Curto and the second named author [CL] gave a complete characterization of hyponormal trigonometric Toeplitz pairs which are pairs of Toeplitz operators with trigonometric polynomial symbols. C. $\mathrm{Gu}[\mathrm{Gu}]$ studied the joint hyponormality of Toeplitz pairs whose coordinates have the same co-analytic parts. The purpose of this article is to provide a complete characterization of hyponormal rational Toeplitz pairs.

Let $\mathcal{H}$ be a complex Hilbert space and let $\mathcal{B}(\mathcal{H})$ be the algebra of bounded linear operators acting on $\mathcal{H}$. For $A, B \in \mathcal{B}(\mathcal{H})$, we let $[A, B]:=A B-B A ;[A, B]$ is the commutator of $A$ and $B$. Given an $n$-tuple $\mathbf{T}=\left(T_{1}, \ldots, T_{n}\right)$ of operators on $\mathcal{H}$, we let $\left[\mathbf{T}^{*}, \mathbf{T}\right] \in \mathcal{B}(\mathcal{H} \oplus \cdots \oplus \mathcal{H})$ denote the self-commutator of $\mathbf{T}$, defined by

$$
\left[\mathbf{T}^{*}, \mathbf{T}\right]:=\left(\begin{array}{cccc}
{\left[T_{1}^{*}, T_{1}\right]} & {\left[T_{2}^{*}, T_{1}\right]} & \ldots & {\left[T_{n}^{*}, T_{1}\right]} \\
{\left[T_{1}^{*}, T_{2}\right]} & {\left[T_{2}^{*}, T_{2}\right]} & \ldots & {\left[T_{n}^{*}, T_{2}\right]} \\
\vdots & \vdots & \ddots & \vdots \\
{\left[T_{1}^{*}, T_{n}\right]} & {\left[T_{2}^{*}, T_{n}\right]} & \ldots & {\left[T_{n}^{*}, T_{n}\right]}
\end{array}\right)
$$

(This definition of self-commutator for $n$-tuples of operators on a Hilbert space was introduced by A. Athavale in [At].) By analogy with the case $n=1$, we shall say ([At], [CMX]) that $\mathbf{T}$ is jointly hyponormal (or simply, hyponormal) if $\left[\mathbf{T}^{*}, \mathbf{T}\right]$ is a positive operator on $\mathcal{H} \oplus \cdots \oplus \mathcal{H}$. Clearly, the hyponormality of an $n$-tuple requires as a necessary condition that every coordinate in the tuple be hyponormal.

Let $\mathbb{T}=\mathbb{R} / 2 \pi \mathbb{Z}$ be the unit circle. The Hilbert space $L^{2} \equiv L^{2}(\mathbb{T})$ has a canonical orthonormal basis given by the trigonometric functions $e_{n}(z)=z^{n}$, for all $n \in \mathbb{Z}$, and the Hardy space $H^{2} \equiv H^{2}(\mathbb{T})$ is the closed linear span of 
$\left\{e_{n}: n=0,1, \ldots\right\}$. An element $f \in L^{2}$ is said to be analytic if $f \in H^{2}$, and co-analytic if $f \in L^{2} \ominus H^{2}$. If $P$ denotes the orthogonal projection of $L^{2}$ onto $H^{2}$, then for every $\phi \in L^{\infty} \equiv L^{\infty}(\mathbb{T})$, the operator $T_{\phi}$ on $H^{2}$ defined by

$$
T_{\phi} g:=P(\phi g) \quad\left(g \in H^{2}\right)
$$

is called the Toeplitz operator with symbol $\phi$. If $\phi$ is a trigonometric polynomial of the form $\phi(z)=\sum_{n=-m}^{N} a_{n} z^{n}$, where $a_{-m}$ and $a_{N}$ are nonzero, then the nonnegative integers $m$ and $N$ denote the co-analytic and analytic degrees of $\phi$, respectively. If $\phi \in L^{\infty}$, write

$$
\phi_{+} \equiv P(\phi) \in H^{2} \quad \text { and } \quad \phi_{-} \equiv \overline{(I-P)(\phi)} \in z H^{2} .
$$

Thus we can write $\phi=\overline{\phi_{-}}+\phi_{+}$. D. Farenick and R. MaEachin [FM] showed that if $U$ is the unilateral shift on $H^{2}$ then the hyponormality of $(U, T)$ implies that $T$ is necessarily a Toeplitz operator. Furthermore they proved that if $\phi=\overline{\phi_{-}}+\phi_{+} \in L^{\infty}$ and if $\psi=\overline{\phi_{-}}+U^{*} \phi_{+} \in L^{\infty}$ then $\left(U, T_{\phi}\right)$ is hyponormal if and only if the single Toeplitz operator $T_{\psi}$ is hyponormal. R. Curto and the second-named author [CL] have studied the hyponormality of $\mathbf{T}=\left(T_{\phi}, T_{\psi}\right)$ when both symbols $\phi$ and $\psi$ are trigonometric polynomials. In [CL], a complete characterization of hyponormal Toeplitz pairs in this case was given. The core of the main result in [CL] is that the hyponormality of $\mathbf{T}=\left(T_{\phi}, T_{\psi}\right)$ (where $\phi$ and $\psi$ are trigonometric polynomials) forces that the co-analytic parts of $\phi$ and $\psi$ necessarily coincide up to a constant multiple, that is,

$$
\phi_{-}=\beta \psi_{-} \quad \text { for some } \beta \in \mathbb{C} .
$$

C. $\mathrm{Gu}[\mathrm{Gu}]$ gave a characterization of hyponormal Toeplitz pairs $\mathbf{T}=\left(T_{\phi}, T_{\psi}\right)$ under the constraint (1.2) on the symbol - the assumption of equal co-analytic parts. In this article we show that if $\phi$ and $\psi$ are rational functions in $L^{\infty}$, then the condition "symbols have equal co-analytic parts" is indeed necessary for the hyponormality of the pair $\mathbf{T}=\left(T_{\phi}, T_{\psi}\right)$ : this follows a spirit of the main result in [CL]. Thus we get a characterization of hyponormal "rational" Toeplitz pairs. A key step for the proof of the main result is accomplished by a direct and careful analysis on the self-commutator of the pair.

The organization of the article is as follows. In Section 2 we introduce basic facts about Toeplitz operators and Hankel operators. In Section 3 we provide auxiliary lemmas to be used in proving the main results. Section 4 is devoted to prove the main results.

Observe that if $\left(T_{1}, T_{2}\right)$ is hyponormal, then so is $\left(T_{1}-\lambda_{1}, T_{2}-\lambda_{2}\right)$ for every $\lambda_{1}, \lambda_{2} \in \mathbb{C}$. Thus if $\phi, \psi \in L^{\infty}$ have Fourier coefficients $\hat{\phi}(n), \hat{\psi}(n)$ for every $n \in \mathbb{Z}$, respectively, then the hyponormality of $\left(T_{\psi}, T_{\phi}\right)$ is independent of the particular values of $\hat{\phi}(0)$ and $\hat{\psi}(0)$. Therefore, throughout the article, we will assume that the 0 -th coefficient $\hat{\phi}(0)$ of the given symbol $\phi$ of a Toeplitz operator is zero. 


\section{Preliminaries}

A bounded linear operator $A$ is called hyponormal if its self-commutator $\left[A^{*}, A\right]:=$ $A^{*} A-A A^{*}$ is positive (semidefinite). Normal Toeplitz operators were characterized by a property of their symbols in the early 1960's by A. Brown and P. Halmos $[\mathrm{BH}]$ and 25 years passed before the exact nature of the relationship between the symbol $\phi \in L^{\infty}$ and the positivity of the self-commutator $\left[T_{\phi}^{*}, T_{\phi}\right]$ was understood (via Cowen's theorem $[\mathrm{Co}]$ ). We shall employ an equivalent variant of Cowen's theorem [Co], that was first proposed by Nakazi and Takahashi [NT].

Cowen's Theorem. For $\phi \in L^{\infty}$, write

$$
\mathcal{E}(\phi):=\left\{k \in H^{\infty}:\|k\|_{\infty} \leq 1 \text { and } \phi-k \bar{\phi} \in H^{\infty}\right\} .
$$

Then $T_{\phi}$ is hyponormal if and only if $\mathcal{E}(\phi)$ is nonempty.

Cowen's method is to recast the operator-theoretic problem of hyponormality for Toeplitz operators into the problem of finding a solution with specified properties to a certain functional equation involving the operator's symbol. This approach has been put to use in many works to study hyponormal Toeplitz operators on the Hardy space of the unit circle.

Let $J$ be the unitary operator on $L^{2}$ defined by $J(f)(z)=\bar{z} f(\bar{z})$. For $\phi \in L^{\infty}$, the operator on $H^{2}$ defined by

$$
H_{\phi} f:=J(I-P)(\phi f) \quad\left(f \in H^{2}\right)
$$

is called the Hankel operator $H_{\phi}$ with symbol $\phi$. If we define the function $\widetilde{v}$ by $\widetilde{v}(z):=\overline{v(\bar{z})}$, then $H_{\phi}$ can be viewed as the operator on $H^{2}$ defined by $\langle z u v, \bar{\phi}\rangle=$ $\left\langle H_{\phi} u, \widetilde{v}\right\rangle$ for all $v \in H^{\infty}$. We write $H_{0}^{2}:=\left\{z f: f \in H^{2}\right\}$ and $\overline{\mathcal{L}}=\{\bar{f}: f \in \mathcal{L}\}$ for $\mathcal{L} \subset L^{2}(\mathbb{T})$. We write, for an inner function $\theta$,

$$
\mathcal{H}(\theta):=H^{2} \ominus \theta H^{2} .
$$

The following is a basic connection between Hankel and Toeplitz operators ([Ni]):

(i) $T_{\phi \psi}-T_{\phi} T_{\psi}=H_{\frac{*}{\phi}}^{*} H_{\psi}\left(\phi, \psi \in L^{\infty}\right)$;

(ii) $H_{\phi} T_{h}=H_{\phi h}=T_{\widetilde{h}}^{*} H_{\phi}\left(h \in H^{\infty}, \phi \in L^{\infty}\right)$.

From this we can see that if $k \in \mathcal{E}(\phi)$ then

$$
\left[T_{\phi}^{*}, T_{\phi}\right]=H_{\bar{\phi}}^{*} H_{\bar{\phi}}-H_{\phi}^{*} H_{\phi}=H_{\bar{\phi}}^{*} H_{\bar{\phi}}-H_{k \bar{\phi}}^{*} H_{k \bar{\phi}}=H_{\bar{\phi}}^{*}\left(1-T_{\widetilde{k}} T_{\widetilde{k}}^{*}\right) H_{\bar{\phi}} .
$$

We here observe that if $\mathbf{T}=\left(T_{\phi}, T_{\psi}\right)$ then the self-commutator of $\mathbf{T}$ can be expressed as:

$$
\left[\mathbf{T}^{*}, \mathbf{T}\right]=\left(\begin{array}{cc}
{\left[T_{\phi}^{*}, T_{\phi}\right]} & {\left[T_{\psi}^{*}, T_{\phi}\right]} \\
{\left[T_{\phi}^{*}, T_{\psi}\right]} & {\left[T_{\psi}^{*}, T_{\psi}\right]}
\end{array}\right)=\left(\begin{array}{ll}
H_{\overline{\phi_{+}}}^{*} H_{\overline{\phi_{+}}}-H_{\overline{\phi_{-}}}^{*} H_{\overline{\phi_{-}}} & H_{\overline{\phi_{+}}}^{*} H_{\overline{\psi_{+}}}-H_{\overline{\psi_{-}}}^{*} H_{\overline{\phi_{-}}} \\
H_{\overline{\psi_{+}}}^{*} H_{\overline{\phi_{+}}}-H_{\overline{\phi_{-}}}^{*} H_{\overline{\psi_{-}}} & H_{\overline{\psi_{+}}}^{*} H_{\overline{\psi_{+}}}-H_{\overline{\psi_{-}}}^{*} H_{\overline{\psi_{-}}}
\end{array}\right) .
$$


A function $\phi \in L^{\infty}$ is said to be of bounded type (or in the Nevanlinna class) if there are functions $\psi_{1}, \psi_{2} \in H^{\infty}(\mathbb{D})$ such that

$$
\phi(z)=\frac{\psi_{1}(z)}{\psi_{2}(z)}
$$

for almost all $z$ in $\mathbb{T}$. Evidently, rational functions in $L^{\infty}$ are of bounded type. We recall ([Ab, Lemma 6]) that if $T_{\phi}$ is hyponormal and $\phi$ is not analytic then $\phi$ is of bounded type if and only if $\bar{\phi}$ is of bounded type. In [Ab, Lemma 3], it was also shown that

$$
\phi \text { is of bounded type } \Longleftrightarrow \operatorname{ker} H_{\phi} \neq\{0\} \Longleftrightarrow \phi=\bar{\theta} b \text {, }
$$

where $\theta$ is an inner function, $b \in H^{\infty}$, and $\theta$ and $b$ are relatively prime, i.e., there does not exist a nonconstant inner function $\omega$ such that $\theta=\omega \theta_{0}$ and $b=\omega b_{0}$ for some $\theta_{0} \cdot b_{0} \in H^{\infty}$. Thus if $f \in H^{2}$ is such that $\bar{f}$ is of bounded type and $f(0)=0$ then we can write

$$
f=\theta \bar{b},
$$

where $\theta$ is an inner function and $b \in \mathcal{H}(\theta)$ satisfies that $b$ and $\theta$ are coprime. In particular, we can easily show that

$$
f=\theta \bar{b} \text { is a rational function } \Longleftrightarrow \theta \text { is a finite Blaschke product. }
$$

Since $T_{\bar{z}} H_{\phi}=H_{\phi} T_{z}$ it follows from Beurling's theorem that ker $H_{\overline{\phi_{-}}}=\theta_{0} H^{2}$ and ker $H_{\overline{\phi_{+}}}=\theta_{+} H^{2}$ for some inner functions $\theta_{0}, \theta_{+}$. If $T_{\phi}$ is hyponormal and $\phi$ is not analytic then by (2.1), $\left\|H_{\overline{\phi_{+}}} f\right\| \geq\left\|H_{\overline{\phi_{-}}} f\right\|$ for all $f \in H^{2}$, so that

$$
\theta_{+} H^{2}=\operatorname{ker} H_{\overline{\phi_{+}}} \subset \operatorname{ker} H_{\overline{\phi_{-}}}=\theta_{0} H^{2},
$$

which implies that $\theta_{0}$ divides $\theta_{+}$, i.e., $\theta_{+}=\theta_{0} \theta_{1}$ for some inner function $\theta_{1}$. Thus if $\phi=\overline{\phi_{-}}+\phi_{+}$is of bounded type, $\phi_{+}(0)=\phi_{-}(0)=0$, and $T_{\phi}$ is hyponormal then we can write

$$
\phi_{+}=\theta_{0} \theta_{1} \bar{a} \quad \text { and } \quad \phi_{-}=\theta_{0} \bar{b}, \quad \text { where } a \in \mathcal{H}\left(\theta_{0} \theta_{1}\right) \text { and } b \in \mathcal{H}\left(\theta_{0}\right) .
$$

On the other hand, as in (2.3), the hyponormality of Toeplitz pairs is also related to the kernels of Hankel operators involved with the analytic and co-analytic parts of the symbol. Indeed it was shown ([Gu, Lemma 6.2]) that if neither $\phi$ nor $\psi$ is analytic and if $\left(T_{\phi}, T_{\psi}\right)$ is hyponormal, then

$$
\operatorname{ker}\left(H_{\overline{\phi_{+}}}\right) \subset \operatorname{ker}\left(H_{\overline{\psi_{-}}}\right) \quad \text { and } \quad \operatorname{ker}\left(H_{\overline{\psi_{+}}}\right) \subset \operatorname{ker}\left(H_{\overline{\phi_{-}}}\right) \text {. }
$$

Throughout the article, whenever writing $f=\theta \bar{a} \in H^{2}$ (where $\theta$ is an inner function and $a \in \mathcal{H}(\theta)$ ), we will assume that $\theta$ and $a$ are relatively prime. 


\section{Auxiliary Lemmas}

To prove the main result we need several auxiliary lemmas. The first two lemmas are technical lemmas about Hankel operators. If $\mathcal{M}$ is a closed subspace of $L^{2}$, we write $P_{\mathcal{M}}$ for the orthogonal projection of $L^{2}$ onto $\mathcal{M}$.

Lemma 1. If $\theta_{0}$ and $\theta_{1}$ are inner functions, then

$$
H_{\overline{\theta_{0} \theta_{1}}}=T_{\widetilde{\theta_{1}}} H_{\overline{\theta_{0}}}+H_{\overline{\theta_{0} \theta_{1}}} P_{\theta_{0} H^{2}} .
$$

Proof. We first claim that $\left.H_{\overline{\theta_{0} \theta_{1}}}\right|_{\mathcal{H}\left(\theta_{0}\right)}=\left.T_{\widetilde{\theta_{1}}} H_{\overline{\theta_{0}}}\right|_{\mathcal{H}\left(\theta_{0}\right)}$. Indeed, if $f \in \mathcal{H}\left(\theta_{0}\right)$ then $\overline{\theta_{0}} f \in \overline{H_{0}^{2}}$, and hence $\bar{z} \theta_{0} \bar{f} \in H^{2}$. Thus

$$
H_{\overline{\theta_{0} \theta_{1}}} f=J(I-P)\left(\overline{\theta_{0} \theta_{1}} f\right)=J\left(\overline{\theta_{0} \theta_{1}} f\right)=\bar{z} \widetilde{\theta_{0}} \widetilde{\theta_{1}} \overline{\widetilde{f}}=T_{\widetilde{\theta_{1}}}\left(\bar{z} \widetilde{\theta_{0}} \overline{\widetilde{f}}\right)=T_{\widetilde{\theta_{1}}} H_{\overline{\theta_{0}}} f .
$$

But since $\operatorname{ker} T_{\widetilde{\theta_{1}}} H_{\overline{\theta_{0}}}=\operatorname{ker} H_{\overline{\theta_{0}}}=\theta_{0} H^{2}$, it follows that

$$
H_{\overline{\theta_{0} \theta_{1}}}=H_{\overline{\theta_{0} \theta_{1}}} P_{\mathcal{H}\left(\theta_{0}\right)}+H_{\overline{\theta_{0} \theta_{1}}} P_{\theta_{0} H^{2}}=T_{\widetilde{\theta_{1}}} H_{\overline{\theta_{0}}}+H_{\overline{\theta_{0} \theta_{1}}} P_{\theta_{0} H^{2}} .
$$

Lemma 2. If $\theta_{0}$ and $\theta_{1}$ are inner functions, then

$$
H_{\overline{\theta_{0} \theta_{1}}} P_{\theta_{0} H^{2}}=P_{\mathcal{H}\left(\widetilde{\theta_{1}}\right)} H_{\overline{\theta_{0} \theta_{1}}} .
$$

Proof. For $f \in H^{2}$, write $f=f_{1}+f_{2}+f_{3}$, where $f_{1} \in \mathcal{H}\left(\theta_{0}\right), f_{2} \in \theta_{0} \theta_{1} H^{2}$ and $f_{3} \in \mathcal{H}\left(\theta_{0} \theta_{1}\right) \ominus \mathcal{H}\left(\theta_{0}\right)$. Thus $f_{3}=\theta_{0} h$ for some $h \in H^{2}$. Observe that

$$
f_{3}=\theta_{0} h \in \mathcal{H}\left(\theta_{0} \theta_{1}\right) \Longleftrightarrow \overline{\theta_{0} \theta_{1}} \theta_{0} h \in \overline{H_{0}^{2}} \Longleftrightarrow h \in \mathcal{H}\left(\theta_{1}\right) .
$$

Therefore we have that

$$
H_{\overline{\theta_{0} \theta_{1}}} P_{\theta_{0} H^{2}} f=H_{\overline{\theta_{0} \theta_{1}}} \theta_{0} h=J(I-P)\left(\overline{\theta_{0} \theta_{1}} \theta_{0} h\right)=J\left(\overline{\theta_{1}} h\right)=\bar{z} \widetilde{\theta_{1}} \overline{\widetilde{h}} .
$$

On the other hand, we have that

$$
\begin{aligned}
P_{\mathcal{H}\left(\tilde{\theta_{1}}\right)} H_{\overline{\theta_{0} \theta_{1}}} f=P_{\mathcal{H}\left(\widetilde{\theta_{1}}\right)} H_{\overline{\theta_{0} \theta_{1}}}\left(f_{1}+f_{3}\right) \\
=P_{\mathcal{H}\left(\widetilde{\theta_{1}}\right)} J(I-P)\left(\overline{\theta_{0} \theta_{1}} f_{1}+\overline{\theta_{1}} h\right) \\
=P_{\mathcal{H}\left(\widetilde{\theta_{1}}\right)} J\left(\overline{\theta_{0} \theta_{1}} f_{1}+\overline{\theta_{1}} h\right) \\
=P_{\mathcal{H}\left(\tilde{\theta_{1}}\right)}\left(\bar{z} \widetilde{\theta_{0}} \widetilde{\theta_{1}} \overline{\widetilde{f_{1}}}\right)+P_{\mathcal{H}\left(\widetilde{\theta_{1}}\right)}\left(\bar{z} \widetilde{\theta_{1}} \overline{\widetilde{h}}\right) \\
=\bar{z} \widetilde{\theta_{1}} \tilde{\widetilde{h}},
\end{aligned}
$$

where the last equality comes from the following observation:

$$
f_{1} \in \mathcal{H}\left(\theta_{0}\right) \Longrightarrow \bar{z} \widetilde{\theta_{0}} \overline{\widetilde{f_{1}}} \in H^{2} \Longrightarrow \bar{z} \widetilde{\theta_{0}} \widetilde{\theta_{1}} \overline{\widetilde{f_{1}}} \in \widetilde{\theta_{1}} H^{2} \Longrightarrow P_{\mathcal{H}\left(\widetilde{\left.\theta_{1}\right)}\right.}\left(\bar{z} \widetilde{\theta_{0}} \widetilde{\theta_{1}} \overline{\widetilde{f_{1}}}\right)=0 .
$$

This completes the proof. 
For a notational convenience we adopt the following notation: If $\phi=\overline{\phi_{-}}+$ $\phi_{+} \in L^{\infty}$ and if $\theta_{1}$ and $\theta_{2}$ are inner functions, we write

$$
\phi_{\theta_{1}, \theta_{2}}:=\overline{P_{H_{0}^{2}}\left(\overline{\theta_{1}} \phi_{-}\right)}+P_{H_{0}^{2}}\left(\overline{\theta_{2}} \phi_{+}\right) .
$$

Thus we have that $\left(\phi_{\theta_{1}, \theta_{2}}\right)_{+}=P_{H_{0}^{2}}\left(\overline{\theta_{2}} \phi_{+}\right)$and $\left(\phi_{\theta_{1}, \theta_{2}}\right)_{-}=P_{H_{0}^{2}}\left(\overline{\theta_{1}} \phi_{-}\right)$. We also abbreviate

$$
\phi_{\theta} \equiv \phi_{\theta, \theta} .
$$

Lemma 3. Let $\phi=\overline{\phi_{-}}+\phi_{+} \in L^{\infty}$ be of the form

$$
\phi_{+}=\theta_{0} \theta_{1} \bar{a} \quad \text { and } \quad \phi_{-}=\theta_{0} \bar{b},
$$

where $\theta_{1}$ and $\theta_{2}$ are inner functions, and $a \in \mathcal{H}\left(\theta_{0} \theta_{1}\right)$ and $b \in \mathcal{H}\left(\theta_{0}\right)$. Then we have:

(i) Suppose $\theta$ is a factor of $\theta_{1}$ and $\omega$ is a factor of $\theta_{0}$. If $T_{\phi}$ is hyponormal then $T_{\phi_{\omega, \theta}}$ is hyponormal. Further if $\omega=1$ then the converse is also true.

(ii) If $\theta$ is an arbitrary inner function and $T_{\phi}$ is hyponormal then $T_{\phi_{\theta}}$ is hyponormal.

Proof. (i) Write $\theta_{1}=\theta \Delta_{1}$ and $\theta_{0}=\omega \Delta_{0}$. If $T_{\phi}$ is hyponormal then by Cowen's theorem there exist a function $k \in H^{\infty}$ with $\|k\|_{\infty} \leq 1$ and a function $h \in H^{2}$ for which $\overline{\theta_{0}} b-k \overline{\theta_{0} \theta_{1}} a=h$, that is, $k a=\theta_{1}\left(b-\theta_{0} h\right)$. Since $a$ and $\theta_{1}$ are relatively prime, it follows that $k=\theta_{1} \zeta$ for some $\zeta \in H^{\infty}$. If we put $k_{1}:=\Delta_{1} \zeta \omega$ then $\left\|k_{1}\right\|_{\infty} \leq 1$ and $\overline{\Delta_{0}} b-k_{1} \overline{\theta_{0} \Delta_{1}} a \in \omega H^{2}$, so that $\overline{P_{H_{0}^{2}}\left(\Delta_{0} \bar{b}\right)}-k_{1} \overline{P_{H_{0}^{2}}\left(\theta_{0} \Delta_{1} \bar{a}\right)} \in H^{2}$, which implies $k_{1} \in \mathcal{E}\left(\phi_{\omega, \theta}\right)$, that is, $T_{\omega, \theta}$ is hyponormal. For the second assertion, observe that if $k \in \mathcal{E}\left(\phi_{1, \theta}\right)$ then $k \theta \in \mathcal{E}(\phi)$.

(ii) Note that if $h \in H^{\infty}$ and $\phi \in L^{\infty}$ then $H_{\overline{P_{H_{0}^{2}}(\bar{h} \phi)}}=H_{\overline{P(\bar{h} \phi)}}=H_{\bar{\phi} h}=$ $H_{\bar{\phi}} T_{h}$. Thus,

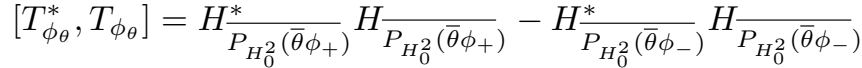

$$
\begin{aligned}
& =H_{\theta \overline{\phi_{+}}}^{*} H_{\theta \overline{\phi_{+}}}-H_{\theta \overline{\phi_{-}}}^{*} H_{\theta \overline{\phi_{-}}} \\
& =T_{\theta}^{*}\left(H_{\bar{\phi}_{+}}^{*} H_{\overline{\phi_{+}}}-H_{\overline{\phi_{-}}}^{*} H_{\overline{\phi_{-}}}\right) T_{\theta} \\
& =T_{\theta}^{*}\left[T_{\phi}^{*}, T_{\phi}\right] T_{\theta},
\end{aligned}
$$

which implies that if $T_{\phi}$ is hyponormal then so is $T_{\phi_{\theta}}$.

The converse of Lemma 3(ii) is not true in general: indeed, a straightforward calculation shows that if $\phi(z)=z^{-2}+3 z^{-1}+2 z+2 z^{2}$ then $T_{\phi}$ is not hyponormal, whereas $T_{\phi_{z}}$ is hyponormal.

Lemma 4. Let $\phi=\overline{\phi_{-}}+\phi_{+} \in L^{\infty}$ and $\psi=\overline{\psi_{-}}+\psi_{+} \in L^{\infty}$ be of the form

$$
\phi_{+}=\theta_{0} \theta_{1} \bar{a}, \phi_{-}=\theta_{0} \bar{b}, \psi_{+}=\theta_{2} \theta_{3} \bar{c} \text { and } \psi_{-}=\theta_{2} \bar{d} \text {, }
$$

where $a \in \mathcal{H}\left(\theta_{0} \theta_{1}\right), b \in \mathcal{H}\left(\theta_{0}\right), c \in \mathcal{H}\left(\theta_{2} \theta_{3}\right)$, and $d \in \mathcal{H}\left(\theta_{2}\right)$. Suppose $\theta$ is an inner function. If $\mathbf{T}=\left(T_{\phi}, T_{\psi}\right)$ is hyponormal then $\mathbf{T}_{\theta}=\left(T_{\phi_{\theta}}, T_{\psi_{\theta}}\right)$ is hyponormal. 
Proof. From the same argument as in the proof of Lemma 3(ii), we have that

$$
\begin{aligned}
{\left[\mathbf{T}_{\theta}^{*}, \mathbf{T}_{\theta}\right] } & =\left(\begin{array}{ll}
{\left[T_{\phi_{\theta}}^{*}, T_{\phi_{\theta}}\right]} & {\left[T_{\psi_{\theta}}^{*}, T_{\phi_{\theta}}\right]} \\
{\left[T_{\phi_{\theta}}^{*}, T_{\psi_{\theta}}\right]} & {\left[T_{\psi_{\theta}}^{*}, T_{\psi_{\theta}}\right]}
\end{array}\right) \\
& =\left(\begin{array}{cc}
T_{\theta}^{*} H_{\overline{\phi_{+}}}^{*} H_{\overline{\phi_{+}}} T_{\theta}-T_{\theta}^{*} H_{\overline{\phi_{-}}}^{*} H_{\overline{\phi_{-}}} T_{\theta} & T_{\theta}^{*} H_{\overline{\phi_{+}}}^{*} H_{\overline{\psi_{+}}} T_{\theta}-T_{\theta}^{*} H_{\overline{\psi_{-}}}^{*} H_{\overline{\phi_{-}}} T_{\theta} \\
T_{\theta}^{*} H_{\overline{\psi_{+}}}^{*} H_{\overline{\phi_{+}}} T_{\theta}-T_{\theta}^{*} H_{\overline{\phi_{-}}}^{*} H_{\overline{\psi_{-}}} T_{\theta} & T_{\theta}^{*} H_{\overline{\psi_{+}}}^{*} H_{\overline{\psi_{+}}} T_{\theta}-T_{\theta}^{*} H_{\overline{\psi_{-}}}^{*} H_{\overline{\psi_{-}}} T_{\theta}
\end{array}\right) \\
& =\left(\begin{array}{cc}
T_{\theta}^{*} & 0 \\
0 & T_{\theta}^{*}
\end{array}\right)\left[\mathbf{T}^{*}, \mathbf{T}\right]\left(\begin{array}{cc}
T_{\theta} & 0 \\
0 & T_{\theta}
\end{array}\right),
\end{aligned}
$$

which implies that if $\mathbf{T}$ is hyponormal then so is $\mathbf{T}_{\theta}$.

If one coordinate of the Toeplitz pair has an analytic symbol then the hyponormality of the pair can be determined by the hyponormality of a single Toeplitz operator (cf. [CL, Theorem 1.10]; [Gu, Theorem 4.1]).

Lemma 5. If $\phi \in H^{\infty}$ is such that $\phi=\theta \bar{a}$ for $a \in \mathcal{H}(\theta)$ and $\psi=\overline{\psi_{-}}+\psi_{+} \in L^{\infty}$ is arbitrary then $\mathbf{T}=\left(T_{\phi}, T_{\psi}\right)$ is hyponormal if and only if $T_{\psi_{1, \theta}}$ is hyponormal.

Proof. See [Gu, Theorem 4.1].

\section{Main Results}

Our main theorem is as follows:

Theorem 1. Let $\phi$ and $\psi$ be rational functions in $L^{\infty}$. If $\mathbf{T}=\left(T_{\phi}, T_{\psi}\right)$ is hyponormal then $\phi-\beta \psi \in H^{2}$ for some constant $\beta$.

Proof. In view of (2.2) and (2.4), we can write

$$
\phi_{+}=\theta_{0} \theta_{1} \bar{a}, \phi_{-}=\theta_{0} \bar{b}, \psi_{+}=\theta_{2} \theta_{3} \bar{c} \text { and } \psi_{-}=\theta_{2} \bar{d},
$$

where the $\theta_{j}$ are finite Blaschke products, $a \in \mathcal{H}\left(\theta_{0} \theta_{1}\right), b \in \mathcal{H}\left(\theta_{0}\right), c \in \mathcal{H}\left(\theta_{2} \theta_{3}\right)$ and $d \in \mathcal{H}\left(\theta_{2}\right)$.

We split the proof into four steps.

STEP 1: If $\theta$ is the greatest common inner factor of $\theta_{1}$ and $\theta_{3}$, then

$$
\mathbf{T}_{1 \theta}=\left(T_{\phi_{1, \theta}}, T_{\psi_{1, \theta}}\right) \text { is hyponormal } \Longrightarrow \mathbf{T}=\left(T_{\phi}, T_{\psi}\right) \text { is hyponormal. }
$$

Proof. We first claim that

$$
\left[T_{\psi}^{*}, T_{\phi}\right]=\left[T_{\psi_{1, \theta}}^{*}, T_{\phi_{1, \theta}}\right]+H_{\overline{\phi_{+}}}^{*} P_{\mathcal{H}(\widetilde{\theta})} H_{\overline{\psi_{+}}} .
$$


Write $\theta_{1}=\theta \delta_{1}$ and $\theta_{3}=\theta \delta_{3}$ for some inner functions $\delta_{1}, \delta_{3}$. Then we have that (4.3)

$$
\begin{aligned}
H_{\phi_{+}}^{*} H_{\overline{\psi_{+}}}= & H_{\overline{\theta_{0} \theta_{1}} a}^{*} H_{\overline{\theta_{2} \theta_{3}} c} \\
= & \left(H_{\overline{\theta_{0} \theta_{1}}} T_{a}\right)^{*}\left(H_{\overline{\theta_{2} \theta_{3}}} T_{c}\right) \\
= & \left(T_{\widetilde{\theta}} H_{\overline{\theta_{0} \delta_{1}}} T_{a}+H_{\overline{\theta_{0} \theta_{1}}} P_{\theta_{0} \delta_{1} H^{2}} T_{a}\right)^{*}\left(T_{\widetilde{\theta}} H_{\overline{\theta_{2} \delta_{3}}} T_{c}+H_{\overline{\theta_{2} \theta_{3}}} P_{\theta_{2} \delta_{3} H^{2}} T_{c}\right) \\
= & T_{a}^{*} H_{\overline{\theta_{0} \delta_{1}}}^{*} T_{\overline{\widetilde{\theta}}} T_{\widetilde{\theta}} H_{\overline{\theta_{2} \delta_{3}}} T_{c}+T_{a}^{*} H_{\overline{\theta_{0} \delta_{1}}}^{*} T_{\widetilde{\widetilde{\theta}}} H_{\overline{\theta_{2} \theta_{3}}} P_{\theta_{2} \delta_{3} H^{2}} T_{c} \\
& \quad+T_{a}^{*} P_{\theta_{0} \delta_{1} H^{2}} H_{\overline{\theta_{0} \theta_{1}}}^{*} T_{\widetilde{\theta}} H_{\overline{\theta_{2} \delta_{3}}} T_{c}+T_{a}^{*} P_{\theta_{0} \delta_{1} H^{2}} H_{\overline{\theta_{0} \theta_{1}}}^{*} H_{\overline{\theta_{2} \theta_{3}}} P_{\theta_{2} \delta_{3} H^{2}} T_{c},
\end{aligned}
$$

where the third equality follows from Lemma 1 . Observe that

$$
T_{a}^{*} H_{\overline{\theta_{0} \delta_{1}}}^{*} T_{\widetilde{\widetilde{\theta}}} T_{\widetilde{\theta}} H_{\overline{\theta_{2} \delta_{3}}} T_{c}=T_{a}^{*} H_{\overline{\theta_{0} \delta_{1}}}^{*} H_{\overline{\theta_{2} \delta_{3}}} T_{c}=H_{\overline{\theta_{0} \delta_{1}} a}^{*} H_{\overline{\theta_{2} \delta_{3}} c}=H_{\overline{\left(\phi_{1, \theta}\right)_{+}}}^{*} H_{\overline{\left(\psi_{1, \theta}\right)_{+}}} .
$$

For each $h \in H^{2}$, if we write $h_{1}:=P_{\mathcal{H}(\theta)} h$ then

$$
T_{\overline{\widetilde{\theta}}} H_{\overline{\theta_{2} \theta_{3}}}\left(\theta_{2} \delta_{3} h\right)=T_{\overline{\widetilde{\theta}}} J(I-P)\left(\overline{\theta_{2} \theta_{3}} \theta_{2} \delta_{3} h\right)=T_{\overline{\widetilde{\theta}}} J\left(\bar{\theta} h_{1}\right)=T_{\overline{\widetilde{\theta}}}\left(\bar{z} \widetilde{\theta h_{1}}\right)=0,
$$

which implies $T_{\overline{\widetilde{\theta}}} H_{\overline{\theta_{2} \theta_{3}}} P_{\theta_{2} \delta_{3} H^{2}}=0$, and hence

$$
T_{a}^{*} H_{\bar{\theta}_{0} \delta_{1}}^{*} T_{\overline{\widetilde{\theta}}} H_{\overline{\theta_{2} \theta_{3}}} P_{\theta_{2} \delta_{3} H^{2}} T_{c}=0 \text {. }
$$

Similarly, we have that $T_{\widetilde{\widetilde{\theta}}} H_{\overline{\theta_{0} \theta_{1}}} P_{\theta_{0} \delta_{1} H^{2}}=0$, and hence $P_{\theta_{0} \delta_{1} H^{2}} H_{\overline{\theta_{0} \theta_{1}}}^{*} T_{\widetilde{\theta}}=0$. Therefore we have that

$$
T_{a}^{*} P_{\theta_{0} \delta_{1} H^{2}} H_{\overline{\theta_{0} \theta_{1}}}^{*} T_{\widetilde{\theta}} H_{\overline{\theta_{2} \delta_{3}}} T_{c}=0 .
$$

On the other hand, by Lemma 2 , we have that

$$
\begin{aligned}
T_{a}^{*} P_{\theta_{0} \delta_{1} H^{2}} H_{\overline{\theta_{0} \theta_{1}}}^{*} H_{\overline{\theta_{2} \theta_{3}}} P_{\theta_{2} \delta_{3} H^{2}} T_{c} & =T_{a}^{*} H_{\overline{\theta_{0} \theta_{1}}}^{*} P_{\mathcal{H}(\widetilde{\theta})} P_{\mathcal{H}(\widetilde{\theta})} H_{\overline{\theta_{2} \theta_{3}}} T_{c} \\
& =H_{\phi_{+}}^{*} P_{\mathcal{H}(\widetilde{\theta})} H_{\overline{\psi_{+}}} .
\end{aligned}
$$

Hence by (4.3)-(4.7), it follows that

$$
\begin{aligned}
{\left[T_{\psi}^{*}, T_{\phi}\right] } & =H_{\overline{\phi_{+}}}^{*} H_{\overline{\psi_{+}}}-H_{\overline{\psi_{-}}}^{*} H_{\overline{\phi_{-}}} \\
& =H_{\overline{\left(\phi_{1, \theta}\right)_{+}}}^{*} H_{\overline{\left(\psi_{1, \theta}\right)_{+}}}+H_{\overline{\phi_{+}}}^{*} P_{\mathcal{H}(\widetilde{\theta})} H_{\overline{\psi_{+}}}-H_{\overline{\left(\psi_{1, \theta}\right)_{-}}}^{*} H_{\overline{\left(\phi_{1, \theta}\right)_{-}}} \\
& =\left[T_{\psi_{1, \theta}^{*}}^{*}, T_{\phi_{1, \theta}}\right]+H_{\overline{\phi_{+}}}^{*} P_{\mathcal{H}(\widetilde{\theta})} H_{\overline{\psi_{+}}}
\end{aligned}
$$

which proves (4.2). Applying (4.2) to $\left[T_{\phi}^{*}, T_{\phi}\right]$ and $\left[T_{\psi}^{*}, T_{\psi}\right]$ gives that

$$
\left[\mathbf{T}^{*}, \mathbf{T}\right]=\left[\mathbf{T}_{1, \theta}^{*}, \mathbf{T}_{1, \theta}\right]+V,
$$

where

But since

$$
V=\left(\begin{array}{ll}
H_{\overline{\phi_{+}}}^{*} P_{\mathcal{H}(\widetilde{\theta})} H_{\overline{\phi_{+}}} & H_{\overline{\phi_{+}}}^{*} P_{\mathcal{H}(\widetilde{\theta})} H_{\overline{\psi_{+}}} \\
H_{\overline{\psi_{+}}}^{*} P_{\mathcal{H}(\widetilde{\theta})} H_{\overline{\phi_{+}}} & H_{\overline{\psi_{+}}}^{*} P_{\mathcal{H}(\widetilde{\theta})} H_{\overline{\psi_{+}}}
\end{array}\right)
$$

$$
V=\left(\begin{array}{ll}
\left(P_{\mathcal{H}(\widetilde{\theta})} H_{\overline{\phi_{+}}}\right)^{*} & 0 \\
\left(P_{\mathcal{H}(\widetilde{\theta})} H_{\overline{\psi_{+}}}\right)^{*} & 0
\end{array}\right)\left(\begin{array}{cc}
P_{\mathcal{H}(\widetilde{\theta})} H_{\overline{\phi_{+}}} & P_{\mathcal{H}(\widetilde{\theta})} H_{\overline{\psi_{+}}} \\
0 & 0
\end{array}\right) \geq 0
$$


it follows that if $\mathbf{T}_{1 \theta}$ is hyponormal then $\mathbf{T}$ is hyponormal.

STEP 2: If $\theta$ is the greatest common inner factor of $\theta_{1}$ and $\theta_{3}$ and if $\omega$ is the greatest common inner factor of $\theta_{0}$ and $\theta_{2}$ then

$$
\mathbf{T}=\left(T_{\phi}, T_{\psi}\right) \text { is hyponormal } \Longrightarrow\left(\mathbf{T}_{\omega}\right)_{1, \theta}=\left(T_{\left(\phi_{\omega}\right)_{1, \theta}}, T_{\left(\psi_{\omega}\right)_{1, \theta}}\right) \text { is hyponormal }
$$

Proof. By Lemma $4, \mathbf{T}_{\omega}=\left(T_{\phi_{\omega}}, T_{\psi_{\omega}}\right)$ is hyponormal. Thus without loss of generality we may assume that $\theta_{0}$ and $\theta_{2}$ are relatively prime. Also by (2.5) we have

$$
\theta_{0} \theta_{1} H^{2} \subset \theta_{2} H^{2} \quad \text { and } \quad \theta_{2} \theta_{3} H^{2} \subset \theta_{0} H^{2} \text {. }
$$

Thus $\theta_{1}=\theta_{2} \varpi_{1}$ and $\theta_{3}=\theta_{0} \varpi_{3}$ for some inner functions $\varpi_{1}, \varpi_{3}$. But since $\theta_{0}$ and $\theta_{2}$ are relatively prime, it follows that

$$
\delta_{1}=\theta_{2} \Delta_{1} \quad \text { and } \quad \delta_{3}=\theta_{0} \Delta_{3} \quad \text { form some inner functions } \Delta_{1}, \Delta_{3} .
$$

Now let $a_{1}:=P_{\mathcal{H}\left(\theta_{0} \delta_{1}\right)} a$ and $c_{1}:=P_{\mathcal{H}\left(\theta_{0} \delta_{3}\right)} c$. Then we have that

$$
\begin{aligned}
& {\left[\mathbf{T}_{1 \theta}^{*}, \mathbf{T}_{1 \theta}\right]=\left(\begin{array}{ll}
{\left[T_{\phi_{1, \theta}}^{*}, T_{\phi_{1, \theta}}\right]} & {\left[T_{\psi_{1, \theta}}^{*}, T_{\phi_{1, \theta}}\right]} \\
{\left[T_{\phi_{1, \theta}}^{*}, T_{\psi_{1, \theta}}\right]} & {\left[T_{\psi_{1, \theta}}^{*}, T_{\psi_{1, \theta}}\right]}
\end{array}\right)} \\
& =\left(\begin{array}{ll}
H_{\overline{\phi_{+}} \theta}^{*} H_{\overline{\phi_{+}} \theta}-H_{\overline{\phi_{-}}}^{*} H_{\overline{\phi_{-}}} & H_{\overline{\phi_{+}} \theta}^{*} H_{\overline{\psi_{+}} \theta}-H_{\overline{\psi_{-}}}^{*} H_{\overline{\phi_{-}}} \\
H_{\overline{\psi_{+}} \theta}^{*} H_{\overline{\phi_{+}} \theta}-H_{\overline{\phi_{-}}}^{*} H_{\overline{\psi_{-}}} & H_{\overline{\psi_{+}} \theta}^{*} H_{\overline{\psi_{+}} \theta}-H_{\overline{\psi_{-}}}^{*} H_{\overline{\psi_{-}}}
\end{array}\right) \\
& =\left(\begin{array}{ll}
H_{\overline{\theta_{0} \delta_{1}} a_{1}}^{*} H_{\overline{\theta_{0} \delta_{1}} a_{1}}-H_{\overline{\theta_{0}} b}^{*} H_{\overline{\theta_{0}} b} & H_{\overline{\theta_{0} \delta_{1}} a_{1}}^{*} H_{\overline{\theta_{2} \delta_{3}} c_{1}}-H_{\overline{\theta_{2}} d}^{*} H_{\overline{\theta_{0}} b} \\
H_{\overline{\theta_{2} \delta_{3}} c_{1}}^{*} H_{\overline{\theta_{0} \delta_{1}} a_{1}}-H_{\overline{\theta_{0}} b}^{*} H_{\overline{\theta_{2}} d} & H_{\overline{\theta_{2} \delta_{3}} c_{1}}^{*} H_{\overline{\theta_{2} \delta_{3}} c_{1}}-H_{\overline{\theta_{2}} d}^{*} H_{\overline{\theta_{2}} d}
\end{array}\right) .
\end{aligned}
$$

Observe that ker $\left[T_{\phi_{1, \theta}}^{*}, T_{\phi_{1, \theta}}\right] \supset \theta_{0} \delta_{1} H^{2}$ and hence $\operatorname{ran}\left[T_{\phi_{1, \theta}}^{*}, T_{\phi_{1, \theta}}\right] \subset \mathcal{H}\left(\theta_{0} \delta_{1}\right)$. Thus $\left[T_{\phi_{1, \theta}}^{*}, T_{\phi_{1, \theta}}\right]$ has the following matrix representation:

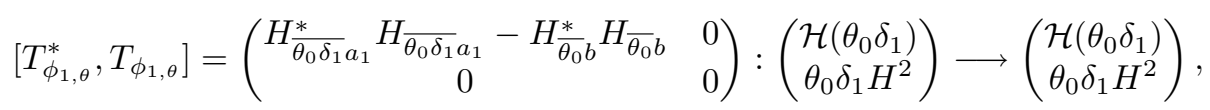

where the left-upper corner will be, without loss in simplifying the notation, understood as the restriction of $H_{\overline{\theta_{0} \delta_{1}} a_{1}}^{*} H_{\overline{\theta_{0} \delta_{1}} a_{1}}-H_{\overline{\theta_{0} b}}^{*} H_{\overline{\theta_{0}} b}$ to $\mathcal{H}\left(\theta_{0} \delta_{1}\right)$. By similar arguments for $\left[T_{\psi_{1, \theta}}^{*}, T_{\phi_{1, \theta}}\right],\left[T_{\phi_{1, \theta}}^{*}, T_{\psi_{1, \theta}}\right]$ and $\left[T_{\psi_{1, \theta}}^{*}, T_{\psi_{1, \theta}}\right]$ using (4.8), we can see that the positivity of $\left[\mathbf{T}_{1 \theta}, \mathbf{T}_{1 \theta}\right]$ is equivalent to the positivity of the restriction, 
say $E$, of it to $\mathcal{H}\left(\theta_{0} \theta_{1}\right) \oplus \mathcal{H}\left(\theta_{2} \theta_{3}\right)$. Note that $E$ can be written as:

$$
\begin{aligned}
E & =\left(\begin{array}{ccccc}
H_{\overline{\theta_{0} \delta_{1}} a_{1}}^{*} H_{\overline{\theta_{0} \delta_{1}} a_{1}}-H_{\overline{\theta_{0}} b}^{*} H_{\overline{\theta_{0}} b} & 0 & H_{\overline{\theta_{0} \delta_{1}} a_{1}}^{*} H_{\overline{\theta_{2} \delta_{3}} c_{1}}-H_{\overline{\theta_{2}} d}^{*} H_{\overline{\theta_{0}} b} & 0 \\
0 & 0 & 0 & 0 \\
H_{\overline{\theta_{2} \delta_{3}} c_{1}}^{*} H_{\overline{\theta_{0} \delta_{1}} a_{1}}^{*}-H_{\overline{\theta_{0}} b}^{*} H_{\overline{\theta_{2}} d} & 0 & H_{\overline{\theta_{2} \delta_{3}} c_{1}}^{*} H_{\overline{\theta_{2} \delta_{3}} c_{1}}^{*}-H_{\overline{\theta_{2}} d}^{*} H_{\overline{\theta_{2}} d} & 0 \\
0 & 0 & 0
\end{array}\right) \\
& :\left(\begin{array}{c}
\mathcal{H}\left(\theta_{0} \delta_{1}\right) \\
\mathcal{H}\left(\theta_{0} \theta_{1}\right) \cap \theta_{0} \delta_{1} H^{2} \\
\mathcal{H}\left(\theta_{2} \delta_{3}\right) \\
\mathcal{H}\left(\theta_{2} \theta_{3}\right) \cap \theta_{2} \delta_{3} H^{2}
\end{array}\right) \longrightarrow\left(\begin{array}{c}
\mathcal{H}\left(\theta_{0} \delta_{1}\right) \\
\mathcal{H}\left(\theta_{0} \theta_{1}\right) \cap \theta_{0} \delta_{1} H^{2} \\
\mathcal{H}\left(\theta_{2} \delta_{3}\right) \\
\mathcal{H}\left(\theta_{2} \theta_{3}\right) \cap \theta_{2} \delta_{3} H^{2}
\end{array}\right),
\end{aligned}
$$

where each entry of $E$ should be understood as a restriction to a suitable subspace. Let

$$
A_{a}:=\left.P_{\mathcal{H}\left(\theta_{0} \theta_{1}\right)} M_{a}\right|_{\mathcal{H}\left(\theta_{0} \theta_{1}\right)} \quad \text { and } \quad A_{c}:=\left.P_{\mathcal{H}\left(\theta_{2} \theta_{3}\right)} M_{c}\right|_{\mathcal{H}\left(\theta_{2} \theta_{3}\right)}
$$

where $M_{x}$ is a multiplication operator with symbol $x$. We now argue that $A_{a}$ and $A_{c}$ are invertible. To see this, suppose $A_{a} f=0$ for some $f \in \mathcal{H}\left(\theta_{0} \theta_{1}\right)$. Then $P_{\mathcal{H}\left(\theta_{0} \theta_{1}\right)}(a f)=0$ and hence $a f \in \theta_{0} \theta_{1} H^{2}$. Since the inner part of $a$ and $\theta_{0} \theta_{1}$ are relatively prime, it follows that $f \in \theta_{0} \theta_{1} H^{2}$. So $f \in \theta_{0} \theta_{1} H^{2} \cap \mathcal{H}\left(\theta_{0} \theta_{1}\right)=\{0\}$, which implies that $A_{a}$ is one-one. But since $A_{a}$ is a finite dimensional operator (because $\theta_{0} \theta_{1}$ is a finite Blaschke product), it follows that $A_{a}$ is invertible. Similarly, $A_{c}$ is also invertible. Observe that

$$
\left.P_{\mathcal{H}\left(\theta_{0} \delta_{1}\right)} A_{a}\right|_{\mathcal{H}\left(\theta_{0} \theta_{1}\right) \cap \theta_{0} \delta_{1} H^{2}}=\left.P_{\mathcal{H}\left(\theta_{0} \delta_{1}\right)} M_{a}\right|_{\mathcal{H}\left(\theta_{0} \theta_{1}\right) \cap \theta_{0} \delta_{1} H^{2}}=0
$$

Thus $A_{a}$ has the following matrix representation:

$$
A_{a}=\left(\begin{array}{cc}
a_{1} & 0 \\
a_{2} & a_{3}
\end{array}\right):\left(\begin{array}{c}
\mathcal{H}\left(\theta_{0} \delta_{1}\right) \\
\mathcal{H}\left(\theta_{0} \theta_{1}\right) \cap \theta_{0} \delta_{1} H^{2}
\end{array}\right) \longrightarrow\left(\begin{array}{c}
\mathcal{H}\left(\theta_{0} \delta_{1}\right) \\
\mathcal{H}\left(\theta_{0} \theta_{1}\right) \cap \theta_{0} \delta_{1} H^{2}
\end{array}\right) .
$$

Since $a_{1}=\left.P_{\mathcal{H}\left(\theta_{0} \delta_{1}\right)} M_{a}\right|_{\mathcal{H}\left(\theta_{0} \delta_{1}\right)}$, we can see, by the same argument as for $A_{a}$, that $a_{1}$ is invertible. As a result, $a_{3}$ is also invertible. Thus the inverse of $A_{a}$ is lower triangular; in fact, $A_{a}^{-1}=\left(\begin{array}{cc}a_{1}^{-1} & 0 \\ -a_{3}^{-1} a_{2} a_{1}^{-1} & a_{3}^{-1}\end{array}\right)$. Similarly, the inverse of $A_{c}$ is also lower triangular. Write

$$
G:=\left(\begin{array}{cc}
A_{a}^{-1} & 0 \\
0 & A_{c}^{-1}
\end{array}\right):\left(\begin{array}{c}
\mathcal{H}\left(\theta_{0} \theta_{1}\right) \\
\mathcal{H}\left(\theta_{2} \theta_{3}\right)
\end{array}\right) \longrightarrow\left(\begin{array}{c}
\mathcal{H}\left(\theta_{0} \theta_{1}\right) \\
\mathcal{H}\left(\theta_{2} \theta_{3}\right)
\end{array}\right) .
$$

Since $G$ is invertible, it follows from (4.9) that

$$
\left[\mathbf{T}_{1 \theta}^{*}, \mathbf{T}_{1 \theta}\right] \text { is positive } \Longleftrightarrow D:=G^{*} E G \geq 0 \text {. }
$$

Since $A_{a}^{-1}$ and $A_{c}^{-1}$ are both lower triangular, $D$ should be of the form

$$
D=\left(\begin{array}{cccc}
d_{1} & 0 & d_{2} & 0 \\
0 & 0 & 0 & 0 \\
d_{3} & 0 & d_{4} & 0 \\
0 & 0 & 0 & 0
\end{array}\right):\left(\begin{array}{c}
\mathcal{H}\left(\theta_{0} \delta_{1}\right) \\
\mathcal{H}\left(\theta_{0} \theta_{1}\right) \cap \theta_{0} \delta_{1} H^{2} \\
\mathcal{H}\left(\theta_{2} \delta_{3}\right) \\
\mathcal{H}\left(\theta_{2} \theta_{3}\right) \cap \theta_{2} \delta_{3} H^{2}
\end{array}\right) \longrightarrow\left(\begin{array}{c}
\mathcal{H}\left(\theta_{0} \delta_{1}\right) \\
\mathcal{H}\left(\theta_{0} \theta_{1}\right) \cap \theta_{0} \delta_{1} H^{2} \\
\mathcal{H}\left(\theta_{2} \delta_{3}\right) \\
\mathcal{H}\left(\theta_{2} \theta_{3}\right) \cap \theta_{2} \delta_{3} H^{2}
\end{array}\right)
$$


On the other hand, we know that

$$
\left[\mathbf{T}_{1 \theta}^{*}, \mathbf{T}_{1 \theta}\right]=\left[\mathbf{T}^{*}, \mathbf{T}\right]-V,
$$

where, for a notational convenience, each term will be understood as a restriction to $\mathcal{H}\left(\theta_{0} \theta_{1}\right) \oplus \mathcal{H}\left(\theta_{2} \theta_{3}\right)$ (Note that $\mathcal{H}\left(\theta_{0} \theta_{1}\right) \oplus \mathcal{H}\left(\theta_{2} \theta_{3}\right)$ reduces $\left[\mathbf{T}^{*}, \mathbf{T}\right],\left[\mathbf{T}_{1 \theta}^{*}, \mathbf{T}_{1 \theta}\right]$ and $V)$. Thus $V$ can be viewed as:

$$
V=\left(\begin{array}{ll}
H_{\overline{\phi_{+}}}^{*} P_{\mathcal{H}(\widetilde{\theta})} H_{\overline{\phi_{+}}} & H_{\overline{\phi_{+}}}^{*} P_{\mathcal{H}(\widetilde{\theta})} H_{\overline{\psi_{+}}} \\
H_{\overline{\psi_{+}}}^{*} P_{\mathcal{H}(\widetilde{\theta})} H_{\overline{\phi_{+}}} & H_{\overline{\psi_{+}}}^{*} P_{\mathcal{H}(\widetilde{\theta})} H_{\overline{\psi_{+}}}
\end{array}\right):\left(\begin{array}{l}
\mathcal{H}\left(\theta_{0} \theta_{1}\right) \\
\mathcal{H}\left(\theta_{2} \theta_{3}\right)
\end{array}\right) \longrightarrow\left(\begin{array}{l}
\mathcal{H}\left(\theta_{0} \theta_{1}\right) \\
\mathcal{H}\left(\theta_{2} \theta_{3}\right)
\end{array}\right)
$$

Observe that

$$
\begin{aligned}
C & :=G^{*} V G \\
& =\left(\begin{array}{cc}
A_{a}^{-1} & 0 \\
0 & A_{c}^{-1}
\end{array}\right)^{*}\left(\begin{array}{ll}
H_{\overline{\phi_{+}}}^{*} P_{\mathcal{H}(\widetilde{\theta})} H_{\overline{\phi_{+}}} & H_{\overline{\phi_{+}}}^{*} P_{\mathcal{H}(\widetilde{\theta})} H_{\overline{\psi_{+}}} \\
H_{\overline{\psi_{+}}}^{*} P_{\mathcal{H}(\widetilde{\theta})} H_{\overline{\phi_{+}}} & H_{\overline{\psi_{+}}}^{*} P_{\mathcal{H}(\widetilde{\theta})} H_{\overline{\psi_{+}}}
\end{array}\right)\left(\begin{array}{cc}
A_{a}^{-1} & 0 \\
0 & A_{c}^{-1}
\end{array}\right) \\
& =\left(\begin{array}{ll}
A_{a}^{-1 *} H_{\overline{\phi_{+}}}^{*} P_{\mathcal{H}(\widetilde{\theta})} H_{\overline{\phi_{+}}} A_{a}^{-1} & A_{a}^{-1 *} H_{\overline{\phi_{+}}}^{*} P_{\mathcal{H}(\widetilde{\theta})} H_{\overline{\psi_{+}}} A_{c}^{-1} \\
A_{c}^{-1 *} H_{\overline{\psi_{+}}}^{*} P_{\mathcal{H}(\widetilde{\theta})} H_{\overline{\phi_{+}}} A_{a}^{-1} & A_{c}^{-1 *} H_{\overline{\psi_{+}}}^{*} P_{\mathcal{H}(\widetilde{\theta})} H_{\overline{\psi_{+}}} A_{c}^{-1}
\end{array}\right) .
\end{aligned}
$$

Since

$$
A_{a}^{-1^{*}} H_{\overline{\phi_{+}}}^{*} P_{\mathcal{H}(\widetilde{\theta})} H_{\overline{\phi_{+}}} A_{a}^{-1}=\left(H_{\overline{\theta_{0} \theta_{1}} a} A_{a}^{-1}\right)^{*} P_{\mathcal{H}(\widetilde{\theta})}\left(H_{\overline{\theta_{0} \theta_{1}} a} A_{a}^{-1}\right)
$$

and

$$
\begin{aligned}
H_{\overline{\theta_{0} \theta_{1}} a} A_{a}^{-1} & =H_{\overline{\theta_{0} \theta_{1}}} T_{a} A_{a}^{-1} \\
& =H_{\overline{\theta_{0} \theta_{1}}} P M_{a}\left(\left.P_{\mathcal{H}\left(\theta_{0} \theta_{1}\right)} M_{a}\right|_{\mathcal{H}\left(\theta_{0} \theta_{1}\right)}\right)^{-1} \\
& =\left.H_{\overline{\theta_{0} \theta_{1}}} P_{\mathcal{H}\left(\theta_{0} \theta_{1}\right)} M_{a}\right|_{\mathcal{H}\left(\theta_{0} \theta_{1}\right)}\left(\left.P_{\mathcal{H}\left(\theta_{0} \theta_{1}\right)} M_{a}\right|_{\mathcal{H}\left(\theta_{0} \theta_{1}\right)}\right)^{-1} \\
& =H_{\overline{\theta_{0} \theta_{1}}},
\end{aligned}
$$

it follows that

(4.12)

$$
\begin{aligned}
A_{a}^{-1^{*}} H_{\overline{\phi_{+}}}^{*} P_{\mathcal{H}(\widetilde{\theta})} H_{\overline{\phi_{+}}} A_{a}^{-1} & =H_{\overline{\theta_{0} \theta_{1}}}^{*} P_{\mathcal{H}(\widetilde{\theta})} H_{\overline{\theta_{0} \theta_{1}}} \\
& =H_{\overline{\theta_{0} \theta_{1}}}^{*} H_{\overline{\theta_{0} \theta_{1}}} P_{\theta_{0} \delta_{1} H^{2}} \quad(\text { by Lemma } 2) \\
& =P_{\mathcal{H}\left(\theta_{0} \theta_{1}\right)} P_{\theta_{0} \delta_{1} H^{2}} \quad\left(\text { since } H_{\bar{\zeta}}^{*} H_{\bar{\zeta}}=P_{\mathcal{H}(\zeta)} \text { for an inner function } \zeta\right) \\
& =P_{\mathcal{H}\left(\theta_{0} \theta_{1}\right) \cap \theta_{0} \delta_{1} H^{2}} .
\end{aligned}
$$

Similarly, we also have that

$$
A_{a}^{-1^{*}} H_{\overline{\phi_{+}}}^{*} P_{\mathcal{H}(\widetilde{\theta})} H_{\overline{\psi_{+}}} A_{c}^{-1}=A_{a}^{-1^{*}} H_{\overline{\theta_{0} \theta_{1}} a}^{*} P_{\mathcal{H}(\widetilde{\theta})} H_{\overline{\theta_{2} \theta_{3} c}} A_{c}^{-1}=H_{\overline{\theta_{0} \theta_{1}}}^{*} P_{\mathcal{H}(\widetilde{\theta})} H_{\overline{\theta_{2} \theta_{3}}} .
$$

Thus, by Lemma 2, we get (4.13)

$$
\begin{aligned}
P_{\mathcal{H}\left(\theta_{0} \delta_{1}\right)} A_{a}^{-1^{*}} H_{\overline{\phi_{+}}}^{*} P_{\mathcal{H}(\widetilde{\theta})} H_{\overline{\psi_{+}}} A_{c}^{-1} P_{\mathcal{H}\left(\theta_{2} \delta_{3}\right)} & =P_{\mathcal{H}\left(\theta_{0} \delta_{1}\right)} H_{\overline{\theta_{0} \theta_{1}}}^{*} P_{\mathcal{H}(\widetilde{\theta})} H_{\overline{\theta_{2} \theta_{3}}} P_{\mathcal{H}\left(\theta_{2} \delta_{3}\right)} \\
& =P_{\mathcal{H}\left(\theta_{0} \delta_{1}\right)} H_{\overline{\theta_{0} \theta_{1}}}^{*} H_{\overline{\theta_{2} \theta_{3}}} P_{\theta_{2} \delta_{3} H^{2}} P_{\mathcal{H}\left(\theta_{2} \delta_{3}\right)} \\
& =0 .
\end{aligned}
$$


Similarly, we also have that

$$
A_{c}^{-1^{*}} H_{\frac{\psi_{+}}{*}} P_{\mathcal{H}(\widetilde{\theta})} H_{\overline{\psi_{+}}} A_{c}^{-1}=P_{\mathcal{H}\left(\theta_{2} \theta_{3}\right) \cap \theta_{2} \delta_{3} H^{2}} .
$$

Therefore by (4.11)-(4.14), $C$ should be of the form

$$
C=\left(\begin{array}{cccc}
0 & 0 & 0 & * \\
0 & 1 & * & * \\
0 & * & 0 & 0 \\
* & * & 0 & 1
\end{array}\right):\left(\begin{array}{c}
\mathcal{H}\left(\theta_{0} \delta_{1}\right) \\
\mathcal{H}\left(\theta_{0} \theta_{1}\right) \cap \theta_{0} \delta_{1} H^{2} \\
\mathcal{H}\left(\theta_{2} \delta_{3}\right) \\
\mathcal{H}\left(\theta_{2} \theta_{3}\right) \cap \theta_{2} \delta_{3} H^{2}
\end{array}\right) \longrightarrow\left(\begin{array}{c}
\mathcal{H}\left(\theta_{0} \delta_{1}\right) \\
\mathcal{H}\left(\theta_{0} \theta_{1}\right) \cap \theta_{0} \delta_{1} H^{2} \\
\mathcal{H}\left(\theta_{2} \delta_{3}\right) \\
\mathcal{H}\left(\theta_{2} \theta_{3}\right) \cap \theta_{2} \delta_{3} H^{2}
\end{array}\right)
$$

In particular, we have that

$$
P_{\mathcal{H}\left(\theta_{0} \delta_{1}\right) \oplus \mathcal{H}\left(\theta_{2} \delta_{3}\right)} C P_{\mathcal{H}\left(\theta_{0} \delta_{1}\right) \oplus \mathcal{H}\left(\theta_{2} \delta_{3}\right)}=0 .
$$

Observe, by (4.10), that $\mathbf{T}_{1 \theta}=\left(T_{\phi_{1 \theta}}, T_{\psi_{1 \theta}}\right)$ is hyponormal if and only if the following operator matrix $\widetilde{D}$ is positive:

$$
\widetilde{D}:=\left(\begin{array}{ll}
d_{1} & d_{2} \\
d_{3} & d_{4}
\end{array}\right):\left(\begin{array}{l}
\mathcal{H}\left(\theta_{0} \delta_{1}\right) \\
\mathcal{H}\left(\theta_{2} \delta_{3}\right)
\end{array}\right) \longrightarrow\left(\begin{array}{l}
\mathcal{H}\left(\theta_{0} \delta_{1}\right) \\
\mathcal{H}\left(\theta_{2} \delta_{3}\right)
\end{array}\right) .
$$

But since by (4.10), (4.11), (4.16), and (4.17),

$$
\begin{aligned}
\widetilde{D} & =P_{\mathcal{H}\left(\theta_{0} \delta_{1}\right) \oplus \mathcal{H}\left(\theta_{2} \delta_{3}\right)} G^{*}\left(\left[\mathbf{T}^{*}, \mathbf{T}\right]-V\right) G P_{\mathcal{H}\left(\theta_{0} \delta_{1}\right) \oplus \mathcal{H}\left(\theta_{2} \delta_{3}\right)} \\
& =P_{\mathcal{H}\left(\theta_{0} \delta_{1}\right) \oplus \mathcal{H}\left(\theta_{2} \delta_{3}\right)}\left(G^{*}\left[\mathbf{T}^{*}, \mathbf{T}\right] G-C\right) P_{\mathcal{H}\left(\theta_{0} \delta_{1}\right) \oplus \mathcal{H}\left(\theta_{2} \delta_{3}\right)} \\
& =P_{\mathcal{H}\left(\theta_{0} \delta_{1}\right) \oplus \mathcal{H}\left(\theta_{2} \delta_{3}\right)} G^{*}\left[\mathbf{T}^{*}, \mathbf{T}\right] G P_{\mathcal{H}\left(\theta_{0} \delta_{1}\right) \oplus \mathcal{H}\left(\theta_{2} \delta_{3}\right)} \\
& \geq 0,
\end{aligned}
$$

it follows that $\mathbf{T}_{1 \theta}$ is hyponormal and this proves (4.1).

STEP 3: We claim that

$$
\theta_{2}=\xi \theta_{0} \quad \text { for some nonzero } \xi \in \mathbb{C} .
$$

Proof. In view of STEP 1, STEP 2, and Lemma 4, we may assume that $\theta_{1}$ and $\theta_{3}$ are relatively prime and $\theta_{0}$ and $\theta_{2}$ are relatively prime. Thus for (4.18) it suffices to show that $\theta_{0}$ and $\theta_{2}$ are constant. Since $\mathbf{T}=\left(T_{\phi}, T_{\psi}\right)$ is hyponormal it follows from again Lemma 4 that $\mathbf{T}_{\theta_{0}}=\left(T_{\phi_{\theta_{0}}}, T_{\psi_{\theta_{0}}}\right)$ is hyponormal. Observe that

$$
\begin{aligned}
& \left(\phi_{\theta_{0}}\right)_{+}=P_{H_{0}^{2}}\left(\overline{\theta_{0}} \theta_{0} \theta_{1} \bar{a}\right)=P_{H_{0}^{2}}\left(\theta_{1} \bar{a}\right)=\theta_{1} \overline{P_{\mathcal{H}\left(\theta_{1}\right)}(a)} \\
& \left(\phi_{\theta_{0}}\right)_{-}=P_{H_{0}^{2}}\left(\overline{\theta_{0}} \theta_{0} \bar{b}\right)=0 \\
& \left(\psi_{\theta_{0}}\right)_{+}=P_{H_{0}^{2}}\left(\overline{\theta_{0}} \theta_{2} \theta_{3} \bar{c}\right) \\
& \left(\psi_{\theta_{0}}\right)_{-}=P_{H_{0}^{2}}\left(\overline{\theta_{0}} \theta_{2} \bar{d}\right)
\end{aligned}
$$


Thus $\phi_{\theta_{0}}$ is analytic. Therefore by Lemma $5, T_{\omega}$ is hyponormal, where

$$
\left.\omega=P_{H_{0}^{2}}\left(\overline{\theta_{1}}\left(\psi_{\theta_{0}}\right)_{+}\right)+\overline{\left(\psi_{\theta_{0}}\right)_{-}}=P_{H_{0}^{2}}\left(\overline{\theta_{1}} P_{H_{0}^{2}}\left(\overline{\theta_{0}} \theta_{2} \theta_{3} \bar{c}\right)\right)+\overline{P_{H_{0}^{2}}\left(\overline{\theta_{0}} \theta_{2} \bar{d}\right.}\right) .
$$

Since $\mathbf{T}=\left(T_{\phi}, T_{\psi}\right)$ is hyponormal we have, by (2.5), that $\theta_{0} \theta_{1} H^{2} \subset \theta_{2} H^{2}$ and $\theta_{2} \theta_{3} H^{2} \subset \theta_{0} H^{2}$. Since $\theta_{0}$ and $\theta_{2}$ are relatively prime we can see that $\theta_{1}=\theta_{2} \Delta_{1}$ and $\theta_{3}=\theta_{0} \Delta_{3}$ for some inner functions $\Delta_{1}$ and $\Delta_{3}$. Observe that

$$
P_{H_{0}^{2}}\left(\overline{\theta_{0}} \theta_{2} \theta_{3} \bar{c}\right)=P_{H_{0}^{2}}\left(\theta_{2} \Delta_{3} \bar{c}\right)=\theta_{2} \Delta_{3} \overline{c_{3}},
$$

where $c_{3}:=P_{\mathcal{H}\left(\theta_{2} \Delta_{3}\right)}(c)$. Since the inner part of $\Delta_{1} c_{3}$ and $\Delta_{3}$ are relatively prime (because $c$ and $\theta_{3}$ are relatively prime) we have that

$$
\omega_{+}=P_{H_{0}^{2}}\left(\overline{\theta_{1}} \theta_{2} \Delta_{3} \overline{c_{3}}\right)=P_{H_{0}^{2}}\left(\overline{\Delta_{1}} \Delta_{3} \overline{c_{3}}\right)=\Delta_{3} \overline{P_{\mathcal{H}\left(\Delta_{3}\right)}\left(\Delta_{1} c_{3}\right)},
$$

where $\Delta_{3}$ and $P_{\mathcal{H}\left(\Delta_{3}\right)}\left(\Delta_{1} c_{3}\right)$ are relatively prime. Since $\theta_{0} d$ and $\theta_{2}$ are relatively prime we also have that

$$
\omega_{-}=P_{H_{0}^{2}}\left(\overline{\theta_{0}} \theta_{2} \bar{d}\right)=\theta_{2} \overline{P_{\mathcal{H}\left(\theta_{2}\right)}\left(\theta_{0} d\right)},
$$

where $\theta_{2}$ and $P_{\mathcal{H}\left(\theta_{2}\right)}\left(\theta_{0} d\right)$ are relatively prime. Therefore the hyponormality of $T_{\omega}$ forces that $\Delta_{3}=\theta_{2} \zeta_{3}$ for some inner function $\zeta_{3}$. Therefore $\theta_{3}=\theta_{0} \Delta_{3}=\theta_{0} \theta_{2} \zeta_{3}$ and hence $\theta_{2}$ is a common inner factor of $\theta_{1}$ and $\theta_{3}$. But since $\theta_{1}$ and $\theta_{3}$ are relatively prime, we must have that $\theta_{2}$ is a constant. Interchanging the roles of $\phi$ and $\psi$ in the above argument gives that $\theta_{0}$ is also a constant. This proves (4.18).

We write $\mathcal{Z}(\theta)$ for the set of all zeros in $\mathbb{D}$ of the inner function $\theta$.

STEP 4: We conclude that

$$
\phi_{-}=\beta \psi_{-}, \quad \text { i.e., } \quad \phi-\beta \psi \in H^{2} \quad \text { for some constant } \beta .
$$

Proof. Suppose $\mathbf{T}=\left(T_{\phi}, T_{\psi}\right)$ is hyponormal. Then $T_{\phi-\beta \psi}$ is hyponormal for all $\beta \in \mathbb{C}$. In view of STEP 3, we may assume that $\theta_{2}=\xi \theta_{0}$ for some $\xi \in \mathbb{C}$. Observe that

$$
\phi-\alpha \psi=\theta_{0} \theta_{1} \theta_{3}\left(\overline{\theta_{3} a}-\xi \alpha \overline{\theta_{1} c}\right)+\overline{\theta_{0}}(b-\bar{\xi} \alpha d) .
$$

We want to show that $b=\beta_{0} d$ for some $\beta_{0} \in \mathbb{C}$. Assume to the contrary that $b \neq \beta d$ for any $\beta \in \mathbb{C}$. Then we can see that $\left(T_{\phi-\beta \psi}, T_{\psi}\right)$ is hyponormal (cf. [Gu, Lemma 5.1]). Let $\beta \in \mathcal{Z}\left(\theta_{0}\right)$, i.e., $\theta_{0}=b_{\beta} \theta_{\beta}$ with $b_{\beta}:=\frac{z-\beta}{1-\bar{\beta} z}$ and some finite Blaschke product $\theta_{\beta}$. Since $\theta_{0}$ and $d$ are relatively prime, it follows that $d(\beta) \neq 0$. Write

$$
\alpha:=\xi \frac{b(\beta)}{d(\beta)} .
$$

Then $\overline{b_{\beta}}(b-\bar{\xi} \alpha d) \in H^{2}$. Thus we have that

$$
(\phi-\alpha \psi)_{-}=\theta_{\beta}\left(\overline{\overline{b_{\beta}}(b-\bar{\xi} \alpha d)}\right) .
$$


But since $\left(T_{\phi-\alpha \psi}, T_{\psi}\right)$ is hyponormal, applying STEP 3 with $\left(T_{\phi-\alpha \psi}, T_{\psi}\right)$ in place of $\left(T_{\phi}, T_{\psi}\right)$ gives that $\theta_{0}$ is an inner factor of $\theta_{\beta}$ up to a unitary constant, a contradiction. Hence $b=\beta_{0} d$ for some $\beta \in \mathbb{C}$, and hence $\phi_{-}=\beta \psi_{-}$for some $\beta \in \mathbb{C}$. This completes the proof of Theorem 1 .

The following corollary is a complete characterization of hyponormal rational Toeplitz pairs.

Corollary 2. Let $\phi=\overline{\phi_{-}}+\phi_{+} \in L^{\infty}$ and $\psi=\overline{\psi_{-}}+\psi_{+} \in L^{\infty}$ be of the form

$$
\phi_{+}=\theta_{0} \theta_{1} \bar{a}, \phi_{-}=\theta_{0} \bar{b}, \psi_{+}=\theta_{2} \theta_{3} \bar{c} \text { and } \psi_{-}=\theta_{2} \bar{d},
$$

where the $\theta_{j}$ are finite Blaschke products, $a \in \mathcal{H}\left(\theta_{0} \theta_{1}\right), b \in \mathcal{H}\left(\theta_{0}\right), c \in \mathcal{H}\left(\theta_{2} \theta_{3}\right)$, and $d \in \mathcal{H}\left(\theta_{2}\right)$. Then $\mathbf{T}=\left(T_{\phi}, T_{\psi}\right)$ is hyponormal if and only if there exists a constant $\beta$ such that

(i) $\phi_{-}=\beta \psi_{-}$;

(ii) $\theta_{3} a-\bar{\beta} \theta_{1} c \in \theta \theta_{0} H^{2}$;

(iii) $T_{\psi \prime}$ is hyponormal, where $\psi^{\prime}:=P\left(\theta \theta_{0} \overline{\theta_{1} c}\right)+\overline{\theta_{2}} d$.

Here $\theta$ is the greatest common inner factor of $\theta_{1}$ and $\theta_{3}$.

Proof. We first assume that $\theta_{1}$ and $\theta_{3}$ are relatively prime. Now suppose $\mathbf{T}=$ $\left(T_{\phi}, T_{\psi}\right)$ is hyponormal. By Theorem $1, \theta_{0}=\theta_{2}$ (up to a unitary constant) and $\phi_{-}=\beta \psi_{-}$for some $\beta \in \mathbb{C}$. Thus $\phi-\beta \psi=\theta_{0} \theta_{1} \theta_{3}\left(\overline{\theta_{3} a}-\beta \overline{\theta_{1} c}\right) \in z H^{2}$. We want to show that $\theta_{0}\left(\overline{\theta_{3} a}-\beta \overline{\theta_{1} c}\right) \in \overline{H^{2}}$. Assume $\theta_{0}\left(\overline{\theta_{3} a}-\beta \overline{\theta_{1} c}\right) \notin \overline{H^{2}}$. Then there exists a nonconstant factor $\zeta_{0}$ of $\theta_{0}$ such that $\phi-\beta \psi=\zeta_{0} \theta_{1} \theta_{3}\left[\theta_{0} \overline{\zeta_{0}}\left(\overline{\theta_{3} a}-\beta \overline{\theta_{1} c}\right)\right]$, where $\overline{\theta_{0}} \zeta_{0}\left(\theta_{3} a-\bar{\beta} \theta_{1} c\right) \in \mathcal{H}\left(\zeta_{0} \theta_{1} \theta_{3}\right)$. By Lemma $5, T_{\widehat{\psi}}$ is hyponormal, where

$$
\widehat{\psi}=\psi_{1, \zeta_{0} \theta_{1} \theta_{3}}=P_{H_{0}^{2}}\left(\theta_{0} \overline{\zeta_{0} \theta_{1} c}\right)+\overline{\theta_{0} \bar{d}} .
$$

Therefore we have that

$$
\operatorname{ker} H_{\overline{\widehat{\psi}_{-}}}=\theta_{0} H^{2} \subseteq \theta_{0} \overline{\zeta_{0}} H^{2} \subset \operatorname{ker} H_{\overline{\hat{\psi}_{+}}},
$$

which is a contradiction (see (2.3)). Thus $\theta_{0}\left(\overline{\theta_{3} a}-\beta \overline{\theta_{1} c}\right) \in \overline{H^{2}}$. Therefore, $\theta_{3} a-$ $\bar{\beta} \theta_{1} c \in \theta_{0} H^{2}$. In particular, $\phi-\beta \psi=\theta_{1} \theta_{3}\left[\theta_{0}\left(\overline{\theta_{3} a}-\beta \overline{\theta_{1} c}\right)\right] \in z H^{2}$, where $\overline{\theta_{0}}\left(\theta_{3} a-\right.$ $\left.\bar{\beta} \theta_{1} c\right) \in \mathcal{H}\left(\theta_{1} \theta_{3}\right)$. Therefore by Lemma $5, T_{\psi^{\prime}}$ is hyponormal, where

$$
\psi^{\prime}:=P\left(\overline{\theta_{1} \theta_{3}} \psi_{+}\right)+\overline{\psi_{-}}=P\left(\theta_{0} \overline{\theta_{1} c}\right)+\overline{\theta_{2}} d=\psi_{1, \theta_{1} \theta_{3}}+c \text { for a constant } c .
$$

The converse is obtained by reversing the above argument.

The proof for the general case can be accomplished by passing to (4.1) with the assumption that $\theta$ is the greatest common inner factor of $\theta_{1}$ and $\theta_{3}$.

By comparison with the cases of trigonometric Toeplitz pairs, we are tempted to guess $\theta_{1}=\theta_{3}$ in the criterion of Corollary 2. If this were true then we would conclude that if $\psi$ is a trigonometric polynomial and $\phi$ is an arbitrary rational 
symbol then the hyponormality of $\mathbf{T}=\left(T_{\phi}, T_{\psi}\right)$ forces $\phi$ to be a trigonometric polynomial. However this is not the case. For example, if $\psi(z)=\frac{1}{6} z^{-1}-z$ and $\phi(z)=\frac{1}{6} z^{-1}+z B \overline{\left(\frac{1}{3} B+\frac{2}{3}\right)}$, where $B=\frac{z-\frac{1}{2}}{1-\frac{1}{2} z}$ then $\phi$ and $\psi$ satisfy all three conditions in Corollary 2 , so that $\mathbf{T}=\left(T_{\phi}, T_{\psi}\right)$ is hyponormal even though $\phi$ is not a trigonometric polynomial.

\section{References}

[Ab] M.B. Abrahamse, Sunormal Toeplitz operators and functions of bounded type, Duke Math. J. 43 (1976), 597-604.

[At] A. Athavale, On joint hyponormality of operators, Proc. Amer. Math. Soc. 103 (1988), 417-423.

[Br] J. Bram, Sunormal operators, Duke Math. J. 22 (1955), 75-94.

[BH] A. Brown and P.R. Halmos, Algebraic properties of Toeplitz operators, J. Reine Angew. Math. 213 (1963/1964), 89-102.

[Con] J.B. Conway, The Theory of Subnormal Operators, Math. Surveys and Monographs, vol. 36, Amer. Math. Soc., Providence, 1991.

[CS] J.B. Conway and W. Szymanski, Linear combination of hyponormal operators, Rocky Mountain J. Math. 18 (1988), 695-705.

[Co] C. Cowen, Hyponormality of Toeplitz operators, Proc. Amer. Math. Soc. 103 (1988), 809-812.

[Cu] R.E. Curto, Joint hyponormality: A bridge between hyponormality and subnormality, Operator Theory: Operator Algebras and Applications (Durham, NH, 1988) (W.B. Arveson and R.G. Douglas, eds.), Proc. Sympos. Pure Math., vol. 51, Part II, Amer. Math. Soc., Providence, (1990), 69-91.

[CL] R.E. Curto and W.Y. Lee, Joint hyponormality of Toeplitz pairs, Memoirs Amer. Math. Soc. 150, No 712 (2001).

[CMX] R.E. Curto, P.S. Muhly and J. Xia, Hyponormal pairs of commuting operators, Contributions to Operator Theory and Its Applications (Mesa, AZ, 1987) (I. Gohberg, J.W. Helton and L. Rodman, eds.), Operator Theory: Advances and Applications, vol. 35, Birkhäuser, Basel-Boston, (1998), 1-22.

[DPY] R.G. Douglas, V.I. Paulsen, and K. Yan, Operator theory and algebraic geometry, Bull. Amer. Math. Soc. (N.S.) 20 (1989), 67-71.

[DY] R.G. Douglas and K. Yan, A multi-variable Berger-Shaw theorem, J. Operator Theory 27 (1992), 205-217.

[FM] D.R. Farenick and R. McEachin, Toeplitz operators hyponormal with the unilateral shift, Integral Equations Operator Theory 22 (1995), 273-280.

[Gu] C. Gu, On a class of jointly hyponormal Toeplitz operators, Trans. Amer. Math. Soc. 354 (2002), 3275-3298.

[McCP] S. McCullough and V. Paulsen, A note on joint hyponormality, Proc. Amer. Math. Soc. 107 (1989), 187-195.

[NT] T. Nakazi and K. Takahashi, Hyponormal Toeplitz operators and extremal problems of Hardy spaces, Trans. Amer. Math. Soc., 338 (1993), 753-769. 
[Ni] N. K. Nikolskii, Treatise on the shift operator, Springer, New York, 1986.

[Xi] D. Xia, On the semi-hyponormal n-tuple of operators, Integral Equations Operator Theory 6 (1983), 879-898.

In Sung Hwang

Department of Mathematics, Sungkyunkwan University, Suwon 440-746, Korea

e-mail: ihwang@skku.edu

Woo Young Lee

Department of Mathematics, Seoul National University, Seoul 151-742, Korea

e-mail: wylee@math.snu.ac.kr 